the researchers' safety concerns, and if valid concerns arise, could make recommendations to the administration for correction of these problems. In addition it may be necessary for the IACUC to send a team to inspect the new surgical suite and make recommendations based on their findings.

Maginnis is Chief, Clinical Medicine Unit, Oregon Regional Primate Research Center, Oregon Health \& Science University, Beaverton, OR.

\section{Managing Resources}

\section{Kimberly S. Edgar, MBA}

The AV's responsibility is to manage the resources of the veterinary care program at the University. This responsibility involves competent management of both the physical plant and the research support service of the veterinary care program. Considering the information presented in this case, the AV's performance falls in the "needs improvement" category. The strategy of exploiting the IACUC's authority to force the large-animal researchers to use the new surgical suite may satisfy short-term objectives, but the trade-off caused by these actions will hinder the ongoing working relationships between the veterinary staff and the researchers.

To resolve these issues and to encourage researchers to use the new surgical suite, the AV must first admit that problems exist and convince the researchers of his desire to foster a better service relationship with them to address future issues more efficiently and effectively, rather than using the IACUC's authority to force decisions. He must be willing to take aggressive actions to learn from these mistakes and try to build a better veterinary care program to meet the needs of the researchers effectively by involving them in resource improvements.

For the physical plant, the AV should immediately establish a team composed of veterinary, research, and facility maintenance staff to examine the numerous operational problems with the new surgical suite. The team should have not only sufficient authority to make decisions but also adequate financial resources to get the surgical suite operating to everyone's comfort level. For example, addressing the complaints with the new surgical monitoring equipment means letting the team explore possible short-term and long-term solutions. One decision may be to arrange with the training coordinator to have service representatives from the monitoring equipment manufacturer come in and do some hands-on training. Another decision may be to have veterinary staff assigned to the surgical suite to do the monitoring during the procedures. Another possibility could be to share the older surgical suite's monitoring equipment with the new suite. Decontaminating the monitoring equipment and transporting it through public corridors may be for health reasons a better short-term solution than transporting large animals.

In regard to the interpersonal issues, the $\mathrm{AV}$ should learn greater sensitivity to the psyches of the researchers and understand the laws of human behavior. The researchers were accustomed to the old surgical suite and felt comfortable with its operation. In the new suite, they are experiencing the natural stressors of surgical procedures compounded by being forced to work in an unfamiliar environment with technologically challenging equipment. Anything unfamiliar or not perfect is going to trigger stressful reactions and result in a desire to retreat back to familiar environments. To address the psychological issues surrounding the new surgical suite, the AV should enlist the veterinary staff to coach the researchers actively through several surgical procedures and to recognize their concerns. As coaches, they should inspire the researchers with encouraging statements and physical assistance to defuse any stressful triggers from escalating during the surgeries. In the role of coaches, they should establish a team of researchers that have successfully used the new suite and promote their accomplishments to the other researchers who are in retreat using the older suite. Peer pressure may influence the recalcitrant researchers to try the new suite again.

Hopefully, this experience has taught the
AV a valuable lesson that successfully managing the veterinary care program requires not only technologically advanced engineering standards for the physical plant resources but also customer-focused performance standards for serving the research community ${ }^{1}$.

\section{Reference \\ 1. Petruzzi, N.C. Business Administration 465: Design and Management of Service System Class Notes. Department of Business Administration, University of Illinois at Urbana-Champaign, Spring Semester, 2003.}

Edgar is Biological Resources Director, The Beckman Institute, University of Illinois, Urbana, IL.

\section{Just Say "No" Joana Visa, DMV, PhD, and Jordi Guinea, PhD}

Adapting to a new situation requires effort, but the advantages usually outweigh the disadvantages. If the research faculty at Great Eastern was informed of the move to the new building and they gave their full support, then the IACUC should not allow them to use the "old" surgery suite for animals housed in the new facility - not only because of the potential risk of disease introduction during animal transport but because of the resulting delay in the conversion of large-animal space to mouse space in the old building.

A possible solution to this problem could be to arrange a meeting with a research faculty to explain the IACUC decision and to provide them with any additional training that they need for using the new equipment. Therefore, it will be necessary to solve the technical problems (surgical lights or the hydraulic table) that had not been recognized during the initial adjustment process as soon as possible.

Visa is Animal Facility Manager, Animal Research Centre, Barcelona Science Park, Barcelona, Spain; Guinea is Animal Facility Manager, Biological School, Barcelona University, Barcelona, Spain. 